PLANT BREEDING

Parasexual Tobacco

by our Botany Correspondent

A FURTher step towards a means of producing hybrid plants without recourse to sexual reproduction has been reported from Brookhaven National Laboratory in New York. Three members of the department of biology have grown viable plants from fused cells of two species of tobacco, Nicotiana glauca and $N$. langsdorffii (P. S. Carlson, H. H. Smith and R. D. Dearing, Proc. US Nat. Acad. Sci., 69, 2292; 1972). Thus they have combined the previous Japanese and British achievements, when isolated protoplasts - the naked contents of plant cells-were induced, on the one hand, to grow into whole plants, and, on the other hand, to fuse with protoplasts from another plant without further development.

The Brookhaven group used similar techniques to obtain and fuse protoplasts from the two species of Nicotiana. Strips of epidermis from young leaves of each species were incubated in an enzyme mixture containing cellulase, macreozyme and sucrose to dissolve away the cell walls. Protoplasts were collected by low speed centrifugation, and then the two species were suspended in sodium nitrate solution, which had previously been shown to induce fusion.

After thirty minutes a peelet was spun down, resuspended and plated on a nutritive medium. At this stage $\mathrm{Dr}$ Carlson and his colleagues had a mixture of fused hybrid protoplasts and unfused parental protoplasts of both species. The methods used to select out the hybrid cells were based on knowledge of the biochemical characteristics of the hybrid $N$. glauca $\times N$. langsdorffii which had already been produced by orthodox sexual means. Regeneration media were used on which only the cells containing the genetic information of both parents would be able to grow.

Out of more than $10^{7}$ protoplasts of each parental species, thirty-three hybrid cells developed into calli which produced rudimentary shoots and leaves but no roots. Further development was obtained by grafting the regenerated shoots onto cut stems of young $N$. glauca plants.

Morphological and biochemical characteristics of the resulting plants, and the flowers and seeds they have now produced, compared exactly with those of the sexual hybrid between the two tobaccos. This confirmed that the parasexual methods used had yielded true hybrids. Of course the next goal in this work is to develop hybrid plants from parents that cannot be mated in the conventional manner, but nevertheless have characters that plant breeders would like to see combined. At present, however, there are technical problems to be overcome. The exact conditions have to be found for the regeneration of the fused protoplasts, and there is also the problem of distinguishing hybrid from parental tissue after growth has been induced. It will be interesting to see the next development in this branch of the new botany.

\section{HISTONES \\ In vitro and in vivo}

from our Cell Biology Correspondent

DURING the past several years a class of RNA molecules which sediment at 7-9S has, with increasing confidence, been claimed to contain messenger RNAs for histone proteins. These putative histone mRNAs have several properties that distinguish them from other putative and identified messengers. For example, they appear to lack the $3^{\prime}$ terminal tracts of polyadenylic acid present in many other messengers; they are transported from the nucleus to the cytoplasm more rapidly than most other RNAs; they appear in small cytoplasmic polysomes only when the cell is replicating its DNA, and they seem to be specified, at least in sea urchin cells, by reiterated and clustered DNA sequences. But, of course, none of these properties of putative histone mRNAs prove that these molecules actually specify histones, which explains why the report of Jacobs-Lorena, Baglioni and Borum (Proc. US Nat. Acad. Sci., 69, 2095; 1972) will be greeted with signs of relief in many quarters. For they have shown quite conclusively that the 7-9S putative histone mRNAs isolated from the polysomes of populations of synchronized HeLa cells in $S$ phase are indeed trans- lated in a cell-free system obtained from mouse ascites tumour cells to yield polypeptides which include all five classes of human histones.

The 7-9S polysomal RNAs extracted from HeLa cells stimulated protein synthesis in the ascites cell-free system from four- to eleven-fold; the histones were identified amongst the products of translation by electrophoresis in SDS and acidic polyacrylamide gels, and by comparing tryptic peptides obtained from the polypeptides made in vitro with those obtained from authentic human histones. Whether or not the 7-9S RNA, as prepared by JacobsLorena et al., contains messengers other than those for histones, and whether or not RNAs other than messengers are present, remains to be seen. The fact that the 7-9S RNA from HeLa cells failed to stimulate appreciably incorporation of tryptophan, an amino-acid absent from histones, suggests that the histone mRNA was not extensively contaminated with mRNAs for other proteins, but the possibility that it contained messengers for a non-histone protein deficient in tryptophan cannot be excluded. The fact that the histone mRNA preparations had less messenger activity in the cell-free system than preparations of haemoglobin mRNA may mean that RNAs lacking messenger activity were present as contaminants, but such differences in messenger activity, which is assayed in vitro, can be explained in several other ways; for example, histone messenger may be more labile than haemoglobin messenger.

Once a histone polypeptide chain has been synthesized, several of its lysine residues may be acetylated, and, as Candido and Dixon report (ibid., 2015), although the sequence of the first

\title{
Selecting Haploid Plant Cells
}

BACTERIA lend themselves to sophisticated genetic analysis for several reasons, not the least of which are that they are haploid and they multiply quickly. Genetic analysis of higher plants and animals, by contrast, is hindered by the much longer life cycle and by the diploid nature of the somatic cells. If somatic cell geneticists could at will produce and maintain haploid cell lines, half their battle would be over, which explains why the report of Gupta and Carlson in next week's Nature New Biology (September 20) should be welcome reading for many.

Parafluorophenylalanine has for the past several years been used occasionally to induce the haploidization of one or two species of different genera of fungi, but Gupta and Carlson appear to be the first people to exploit this com- pound to select and maintain cultures of haploid cells from higher plants. As a model system they established in culture cells of haploid and diploid strains of tobacco plants, and then added various concentrations of parafluorophenylalanine to the culture media. At concentrations which kill a callus of diploid cells a haploid cell callus continues to grow vigorously. It should therefore be possible to use this amino-acid to select haploid cells from populations of cells of varying ploidy, such as emerge in anther cultures. To date, of course, Gupta and Carlson have used only tobacco plant cells, but if the effect of parafluorophenylalanine is not specific to this species they may well have hit upon a generally useful method of obtaining haploid, higher plant, cell cultures. 
twenty-five amino terminal amino-acids of trout testis histone III is identical to the sequence of the corresponding segment of calf thymus histone III, the pattern of acetylation of these two histones differs. In both calf and trout histone III, lysine residues 14 and 23 are acetylated but the lysine residues at positions 9 and 18 in some of the trout testis histone molecules are also acetylated.

The first twenty-two amino-acids in trout testis histone $\mathbf{I I} \mathbf{b}_{2}$, which differs at several positions from the amino-acids of the corresponding segment of calf thymus histone $\mathrm{II}_{2}$, include four acetylated lysine residues, those at positions $5,10,13$ and 18 . Comparison of the amino-acids adjacent to acetylated lysine residues in trout testis histones III and IIb $b_{2}$ reveals an interesting pattern. Acetylated Iysine residues are either bordered on either side by aminoacids with short neutral side chains (glycine, threonine and alanine, the codons of which are related by single base changes) or the acetylated lysine residue is part of a Lys-Arg, Arg-Lys or Lys-Lys pair. Such sequences may well, therefore, provide part of the recognition signals which presumably dictate the sites at which specific acetylase can act. But, as Candido and Dixon point out, other factors such as the secondary structures of these proteins must be involved in the specificity of acetylation because, for example, lysine residue 47 in rabbit and calf histone $\mathrm{I}$ is not acetylated, even though it is preceded by a threonine residue and followed by an alanine residue.

Allfrey and Mirsky, Dixon and others, have postulated that the acetylation, methylation and phosphorylation of histones alter the ability of these proteins to react with DNA, and thereby play a part in the regulation of gene expression. The observation, made by Ryan and Cristofalo (Biochem. Biophys. Res. Commun., 48, 735; 1972), that as populations of cultivated human fibroblasts (WI-38 cells) age, the rate at which the cells are able to acetylate their histones decreases markedly, even though the ratio of histone/DNA per cell remains constant, can, of course, be accommodated by this hypothesis. But whether changes in the extent or pattern of acetylation of histones have any role in ageing remains to be seen.

\section{MEMBRANES \\ Solid for Liquidity}

from our Molecular Biology Correspondent Considering the weight of effort that has been brought to bear on the analysis of line shapes in n.m.r. and e.s.r. spectra of phospholipid bilayers, and the minute detail concerning the static and dynamic characteristics of the chains in these

bilayers that has been extracted, one might suppose that the point of diminishing returns has long been passed. All the same, another squeeze of the lemon has produced something new. Work from two laboratories has demonstrated that, depending on conditions, there is rapid translational diffusion of phospholipid molecules in the plane of the bilayer. Since the now celebrated demonstration by Frye and

\section{Plate Tectonics in South-east Asia}

As Tarling remarked in Nature (239, 38 ; 1972) two weeks ago, South-east Asia is a complex area which is little known from the point of view of plate tectonics. But with the publication of next Monday's Nature Physical Science (September 18) the situation will have been transformed by a magnificent interpretation of the tectonic development of eastern Indonesia in relation to the breakup of Gondwanaland, carried out by Audley-Charles, Carter and Milsom of Imperial College, London. For what Audley-Charles and his colleagues have done is to marshal both geological and geophysical evidence for the evolution of Indonesia from the early Cretaceous to the present. Of course, only time will tell whether the interpretation is correct in detail; but what is certain is that the study by AudleyCharles et al. will for some time to come be regarded as the basic model against which detail will be judged.

The problem with South-east Asia stems from the complex geological history of the Banda Arcs (see map)the Inner Banda Arc, which extends from the volcanic islands east of Flores through Wetar to the volcanoes in the Banda Sea south of Seram, and the Outer Banda Arc, which extends from Timor through Tanimbar, $\mathrm{Kai}$ and Seram to Buru. In general terms,
Edidin of protein antigens diffusing rapidly across a cell membrane, this result should perhaps occasion little surprise. Its demonstration, together with an evaluation of diffusion coefficients, is nevertheless of no little interest, and reinforces again the view, asserted in recent years, that real and model membrane systems in general behave as layers of fluid, within which proteins may float more or less freely.
Audley-Charles et al. conclude that the Outer Banda Arc formed part of the Australian continental margin from at least the Permian, but that Seram, Buru and eastern Sulawesi became detached after the breakup of Gondwanaland-a conclusion which implies that these areas have undergone very large rotations, and that during the mid-Mesozoic central New Guinea must have lain to the east of Australia.

The subsequent history of the area, the geological manifestation of which is a series of orogenic events recorded in the Outer Banda Arc and eastern Sulawesi, is then seen in terms of a series of collisions between the northward-drifting Australian continent and a series of northward-dipping subduction zones. The first such collision took place between the late Cretaceous and the Eocene, after which renewed subduction began further north to allow the continuing northward motion of Australia. The second collision (with the new subduction zone) then occurred during the middle Miocene, by which time central New Guinea had separated from the east coast of Australia and had rotated into an east-west position. There then developed another subduction zone still further north, with which the Australian continent collided (the third collision) during the late Pliocene.

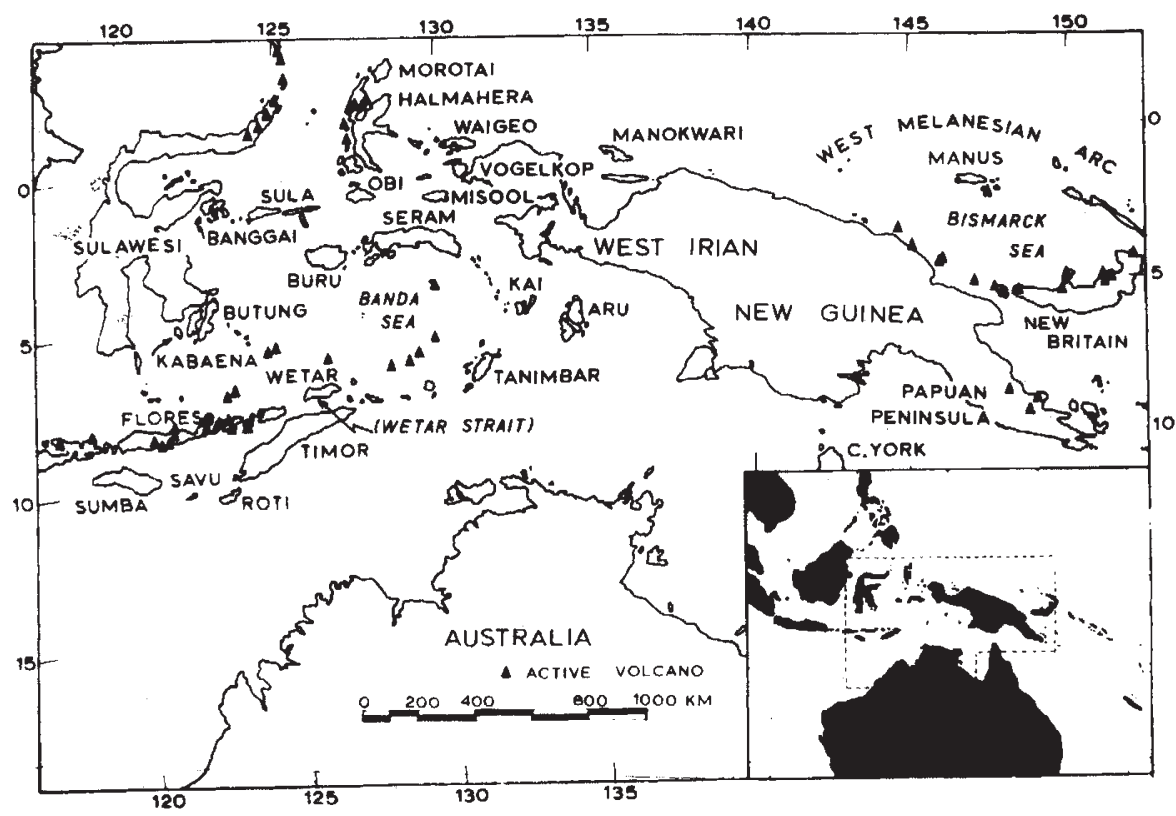

